\title{
A comparison of two Detailed Clinical Model representations:
}

\section{FHIR and CDA}

\author{
Marten Smits ${ }^{1}$, Ewout Kramer ${ }^{1}$, Martijn Harthoorn ${ }^{1}$, Ronald Cornet ${ }^{2,3}$ \\ ${ }^{1}$ Software Engineering Department, Furore, The Netherlands \\ ${ }^{2}$ Department of Medical Informatics, Academic Medical Center - University of Amsterdam, The Netherlands \\ ${ }^{3}$ Department of Biomedical Engineering, Linköping University, Sweden
}

\begin{abstract}
Introduction:In recent years several standardized modeling methods have been proposed that separate health related data models from their underlying technical data model. These methods presuppose representation of information independently of (or uninfluenced by) technical considerations. Among these methods is the Detailed Clinical Model (DCM) paradigm. One of the pillars of this paradigm is that all representations convey the same meaning and are independent of the technical standard that is used and the DCM standard claims to achieve that. In this paper we will challenge that claim by modeling the specific DCMs in two different technical standards (CDA and FHIR) and testing if messages based on these models are interconvertible.

Methods: We identified and categorized the problems that may arise when mapping or combining multiple standards creating representations of selected DCMs in both FHIR and CDA to determine possible fundamental problems using a technology independent model (DCM) to represent technical models (FHIR and CDA).
\end{abstract}

\section{Correspondence to:}

Marten Smits, MSc

Furore

Address: Bos en Lommerplein 280, 1055RW, Amsterdam

E-mail: m.smits@furore.com
To test if the theoretical problems we encountered while creating our example messages also occur during the actual transformation, and to determine any additional problems, we attempted to transform the Clinical Document Architecture (CDA) representations of the DCMs to the FHIR representations using Extensible Style sheet Language Transformations (XSLT).

Results: Most aspects of the DCMs could be properly represented in both FHIR and CDA, and can be transformed from CDA to FHIR. However, we identified fundamental issues where information was lost or its meaning was changed. This results in fundamental difficulties during the implementation of the standards and when transforming one standard to another.

Conclusion: Our research shows that possible loss and change of meaning and lack of interconvertibility occurs when implementing two separate technical standards based on the same DCMs. This indicates that it does matter which technical standard is used to implement a DCM.

\section{Keywords}

Knowledge Representation; Electronic Health Records; Detailed Clinical Models

\section{EJBI 2015; 11(2):en7-en17}

received: October 14, 2014

accepted: January 6, 2015

published: January 20, 2015

\section{Introduction}

\subsection{Context}

Hospitals and other healthcare providing organizations typically have many different computer systems for everything from billing records to patient tracking. This software must be able to communicate with other software inside and outside the facility to share clinical informa- tion. It is important for hospitals and patient safety in general that exchanged information is not lost or altered.

There is general agreement that making clinical documentation uniform saves time and resources and the exchange of data between healthcare institutions is much simpler when there are less different data definition standards. For research and healthcare quality indicators it is also desirable that healthcare data is saved in a uniform way and close to the health processes. [1]

The eight academic hospitals in the Netherlands and the National IT Institute for Healthcare in the Nether- 
lands (Nictiz), a knowledge center for IT and innovation in the healthcare sector, took the initiative to work together on standardization of health data. The project Generic Data for Patient Transfers (GenOGeg - "Generieke OverdrachtsGegevens" in Dutch), was started in January 2012 as the first project of the collaboration. The scope of the project is to create a national cross-specialty exchange dataset for when a patient gets transferred between healthcare facilities. [1]

In order to secure corresponding data, the Detailed Clinical Model (DCM) paradigm is chosen, and a sizable set of DCMs have been published as a result of this project. DCMs provide a method to specify what information is potentially relevant. DCMs combine terminology, professional knowledge, and data specification into information models, from which various technical solutions can be developed. 2 Nictiz is planning to use a Health Level Seven (HL7) version 3 Clinical Document Architecture (CDA) Release 2 standard based on the DCMs as a solution for this exchange.

Meanwhile, developments of a next version of HL7 are ongoing, coined Fast Health Interoperable Resources (FHIR), which can also be used to represent DCMs. Over the years, a lot of effort has been put in the specification of clinical data elements. Clinicians, regulatory agencies, health statisticians, institutions for quality control and others invest in clinical data standards. [3] With this growth of standards, the demand of standards that can exchange information with other standards also grows.

As the GenOGeg project constitutes a large effort from many people and different organizations, it is relevant to consider how this work will be able to cope with the inevitable change in landscape of data standards in health. For this it is vital to establish whether the current chosen implementation of DCM can be transformed into the next generation of standards.

\subsection{Research}

As FHIR is based on other models than HL7v3 4, we aim to investigate to what extent CDA representations of the GenOGeg DCMs are different from the FHIR representations and which problems occur when transforming one into the other. A review about detailed clinical models states:

"DCMs organize health information via combining knowledge, data element specification, relationships between data elements, and terminology into information models that allow deployment in different technical formats." [5]

The research question of this article is: Do different representations of DCMs convey the same meaning and are DCMs independent of the technical standard that is used?

Sub questions are:

- What problems arise through conceptual analysis (creating and comparing example messages)?
- What problems does practical XSLT transformation add?

\section{Background}

\subsection{Detailed Clinical Models}

The Detailed Clinical Model methodology is a draft ISO standard (ISO/PRF TS 13972). It is used to describe a technology-agnostic data model and narrative around interpretation of the model and the data. The methodology was created to standardize the way data is modeled.

DCMs describe the structure of the clinical data that is stored in electronic patient records, sent between clinical systems, and referenced in decision support rules. DCMs also describe the line between the terminology model and the information model, which is, just like defining value sets, helpful for a compatible exchange of data. A DCM is a relatively small model, designed to express a clinical concept in a standardized and reusable way. Data elements and attributes of a clinical concept, the possible values and types, and relationships needed to convey the clinical reality are described by a DCM in a way that is readable to both modelers and clinicians. 5.

\subsection{HL7 v3 CDA release 2}

The HL7v3 Clinical Document Architecture (CDA) is a standard for exchanging and saving medical documents. A typical CDA document would be an admission report, discharge summary, imaging report etc. CDA uses XML, although it allows for a non-XML body (pdf, Word, jpg etc.) for simple implementations.

CDA Release 2, based on the HL7v3 Reference Information Model (RIM), basically consists of tags, which harbor the semantics for persons and document properties that can be used to describe the structure and the hierarchy of the document.

CDA release 2 was launched in 2005 and has gained much popularity internationally. The popularity comes from the simplicity of the model. The fact that the model is generic and is not bound to any domain means there is a lot of freedom during implementation. Persistence, the ability to sign documents, context, and human readability are all characteristics of the model that is defined in CDA.

\subsection{FHIR}

FHIR is a new draft standard based on emergent industry approaches. FHIR claims to combine the best features of the previous HL7 standards while being fast and easy to implement. [6] The FHIR standard can be used as a stand-alone data exchange standard, but can also be used in partnership with existing widely used standards. [7] The basic building block of a FHIR document is a resource. An example of a patient resource can be found in Figure 1 . 
Resources have a wide range of uses, from clinical content such as care plans and diagnostic reports through infrastructure such as Message Header and conformance statements. 7] Resources define all exchangeable content, despite the fact they are used in totally different fashions, they all share the following set of characteristics:

- A common way to define and represent them, building them from data types. that define common reusable patterns of elements.

- A common set of metadata.

- A human-readable part.

FHIR's philosophy is to build documents from a set of resources that, either by themselves or when combined, satisfy the majority of common use cases. Extensions can be used to cover the remaining content as needed. Usually, specific use cases are implemented by combining resources through the use of resource references. [7]

\section{Methods}

To challenge the claim of compatibility of two representations of the Detailed Clinical Models, we chose CDA for being the most frequent implementation and FHIR for being its most likely successor. To determine the presence of fundamental and less severe problems which could occur when representing a technology-independent model (DCM) in a technical model (FHIR and CDA), we created example messages based on the GenOGeg's DCMs in both representations (FHIR and CDA).

To test if the theoretical problems we determined while creating our example messages also occur during the actual transformation, we attempted to transform the CDA representations to the FHIR representations of the DCMs using Extensible Stylesheet Language Transformations (XSLT).

A visual representation of the methods is depicted in Figure 2

\subsection{Creating example messages}

To establish the presence of problems which could occur when trying to fit a technical model (FHIR and CDA) onto a technology independent model (DCM), we created example messages based on the GenOGeg's DCMs.

These DCMs are particularly suitable for this research because they are widely accepted, describe a representative set of data, and are the first DCMs on a national cross-specialty level.

We made a selection of the DCMs, where we expected most problems would arise. We selected the DCMs due to their amount of complexity as opposed to the ones we did not select. The DCMs and the reason for their selection can be found in Table 1 .

DCMs are technology-independent models that are not bound to any technical standard and have already de- fined the clinical concepts in a standardized and reusable way. Therefore we used DCMs as a starting point and attempted to fit them into two technical models (FHIR and CDA). To overcome the fact that FHIR is still in Draft Standard for Trial Use (DSTU), we adjusted our representations to the latest version of FHIR when revisions were made to the FHIR standard during our study.

We will describe here how we used the implementation guide of GenOGeg's American equivalent, the Consolidated Clinical Document Architecture (C-CDA), as the implementation guide of the GenOGeg project was not yet finished.

The implementation guide describes how to model document body entries and provides example messages, which we remodeled to represent a DCM. Each part of each DCM was mapped to corresponding document body entries described in the C-CDA implementation guide. We used XML schema validation to check the validity of our messages. The purpose of an XML Schema is to define the legal building blocks of a specific XML document (for example FHIR) and validate if an XML message is in accordance with these building blocks.

As CDA is a rather flexible standard, it is more than likely that different modelers create different CDA representations based on the same DCMs. To prevent outcome bias due to keeping FHIR in mind when creating our CDA representations, we used the rules and CDA subset of the C-CDA.

To create the FHIR example messages for the DCMs, we started by studying the FHIR specification [8]. In the specification, examples were provided, which we used as a template to create our messages. From the list of resources we selected the resource that corresponded with the DCM model, which could be retrieved from the FHIR specification.

We remodeled the examples so they conformed to the rules of a DCM representation. To check the validity of the XML document of our newly created example messages we used the FHIR-atom XML schema validation.

\subsection{Comparing example messages to identify discrepancies}

From the start of example message creation, we kept an inventory of problems. We documented the problems we had when representing a DCM using FHIR and CDA. With each step we took in the process we assessed whether the example message was still in accordance with the DCM or that change or loss of meaning occurred with the last adjustment.

We compared the semantics of the FHIR and CDA example messages on each element and identified and categorized the differences between both representations. The initial categorization was based on problems described in literature [3, 9, 10, 11], and consisted of: Problems with coded values; Difference in Relational Structures / Hierarchies; Difference in requirements and restrictions; Use 
of narratives. For problems that could not be categorized additional categories were introduced.

\subsection{Transforming the CDA example messages to FHIR}

To test if the theoretical problems while creating our example messages also occur during actual implementation, and to identify if any additional problems arise, we attempted to transform the CDA representations to the FHIR representations of the DCMs using XSLT. XSLT is a language for transforming XML documents into other XML documents. [12] The input and the output for XSLT are the same type of objects (both XML). This has immediate benefits: for example it is possible to do a complex transformation as a series of simple transformations, and it is possible to do transformations in either direction using the same technology. 13

During the transformation of each CDA example message we assessed whether we had to remove, add or change information to come to a valid FHIR message, which we validated using the FHIR-atom XML schema.

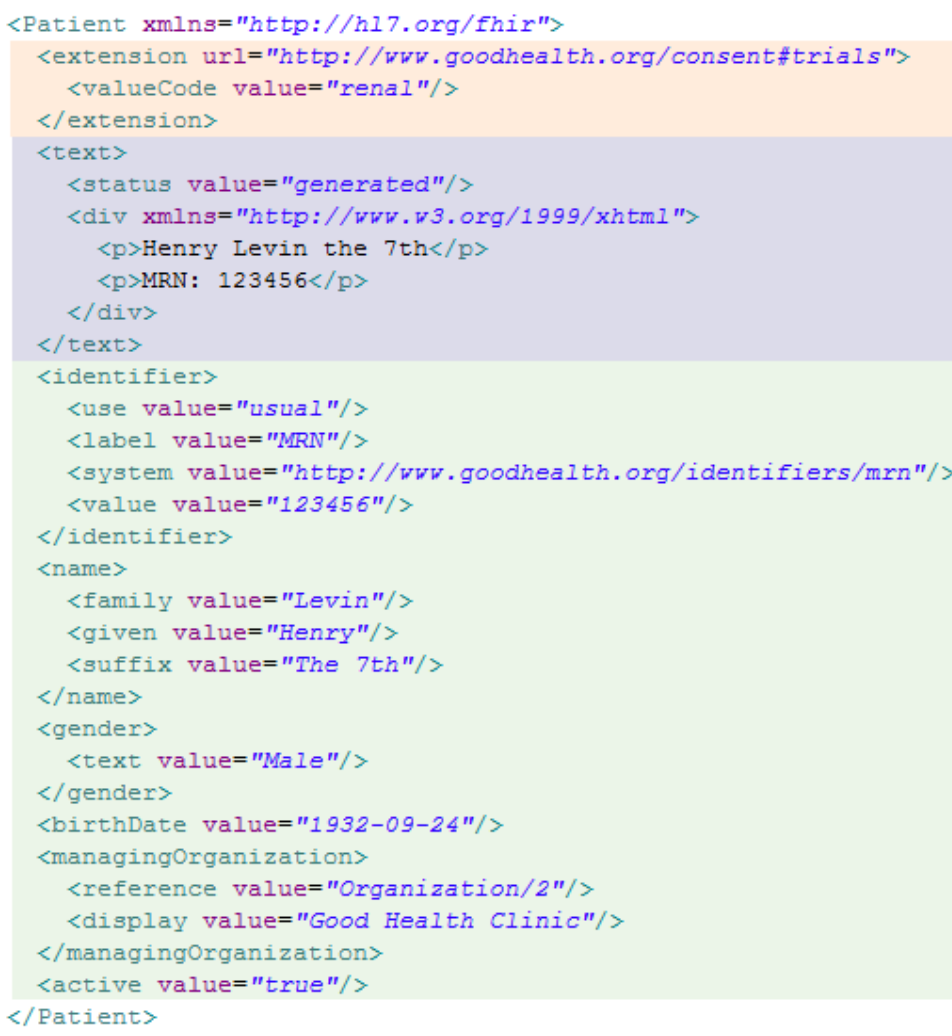

Figure 1: Example of a patient resource [6].

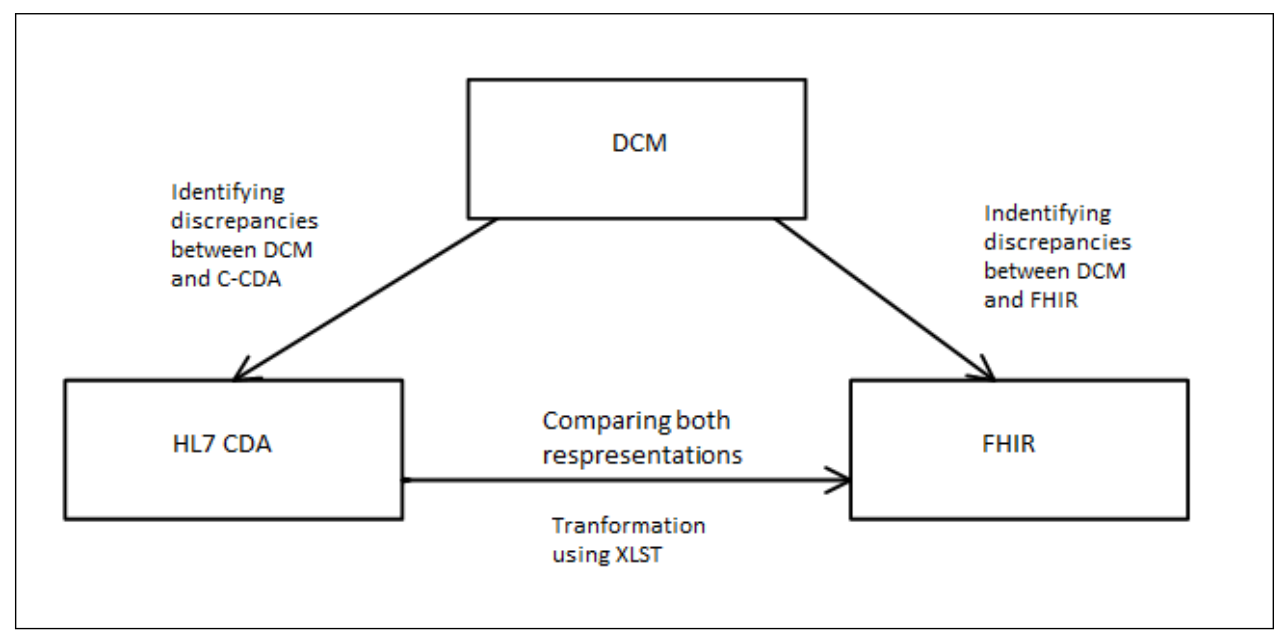

Figure 2: Visual representation of the methods. 
Table 1: Selected DCMs, with rationale for their use.

\begin{tabular}{ll}
\hline DCM & Reason for selection \\
\hline Alert & Ambiguous definitions, expected mismatch between technical models \\
Barthel Index & To compare support for scores and composite observations \\
Family History & Possible relationship problems \\
Lab Report & Hierarchy of report/section/observation, coding systems, work-ow data elements \\
Medication & Definition and scope of major components of the medication workflow \\
Patient & Because it's needed for the CDA header \\
Plan of Care & Complex, scope, tension between free text/structured representations \\
\hline
\end{tabular}

All problems and proposed solutions we encountered along the way were documented.

\section{Results}

The problems we encountered are each described in a separate section. In these sections we describe whether we encountered the problems during the creation of our representations of the DCM, the transformation of the CDA representation to FHIR, or both. All FHIR and CDA representations and XSLT files used for the transformation from CDA to FHIR can be found on Github (https: //gist.github.com/mmsmits/57e027d5435d678b95ad).

Table 2 shows the category of the problems we encountered, where we encountered them, whether they are described in the literature, and in which section they are described.

\subsection{Coded values}

We encountered differences in the use of coded values in DCMs, FHIR, and CDA. Coded values can be used to define observations (e.g. SNOMED CT codes), but also for other purposes, e.g. to give a care plan a status.

As shown in Table 3 , the status codes in DCMs and in FHIR differ significantly.

- Planned: The plan is in development or awaiting use but is not yet intended to be acted upon.

- Active: The plan is intended to be followed and used as part of patient care.

- Completed: The plan is no longer in use and is not expected to be followed or used in patient care.

These codes are not fully translatable to each other; "new" can be mapped onto to "planned", but one cannot map "cancelled" and "aborted" to "completed".

To represent that a care plan is "ordered", the DCM and CDA both use a CCD code, whereas FHIR uses a different resource with a subject that refers to the actual care plan. This order resource requires information that CDA cannot provide.

Another problem occurred when we modeled the normal ranges of the different lab results. In C-CDA the nor- mal range of Hemoglobin is modeled as shown in Figure 3

It specifies the normal ranges for males and females in semi-structured text. In FHIR the same is modeled fully structured, as shown in Figure 4.

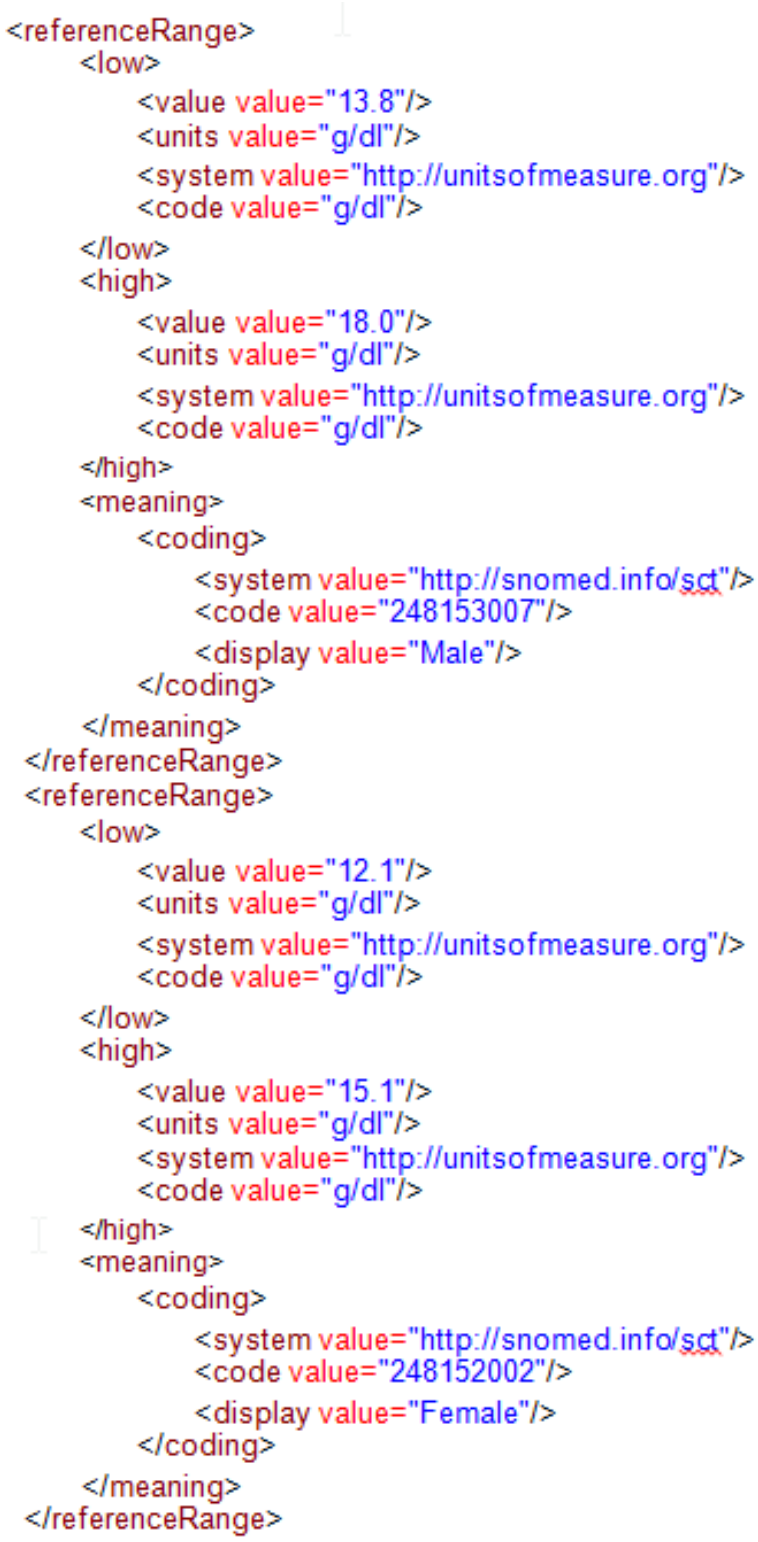

Figure 4: A normal range of hemoglobin represented according to the FHIR specification. 
Table 2: List of encountered problems.

\begin{tabular}{lccc}
\hline Problem & Representations & Transformation & Literature \\
\hline Coded values & $\mathrm{X}$ & $\mathrm{X}$ & $\mathrm{X}$ \\
Difference in Relational Structures / Hierarchies & $\mathrm{X}$ & $\mathrm{X}$ & $\mathrm{X}$ \\
Requirements and restrictions & $\mathrm{X}$ & $\mathrm{X}$ & $\mathrm{X}$ \\
Narrative & & $\mathrm{X}$ & $\mathrm{X}$ \\
Null avors and negation indicators & & $\mathrm{X}$ & \\
Meaning of attributes & $\mathrm{X}$ & \\
\hline
\end{tabular}

Table 3: Care plan status codes in DCM and FHIR.

\begin{tabular}{ccc}
\hline DCM Concept Name & DCM Concept Code & FHIR Code \\
\hline Ordered & New & Planned \\
Requested & New & Planned \\
Pending & Active & Active \\
In Process & Active & Active \\
On hold & Held & $? ?$ \\
Cancelled & Cancelled & Completed \\
No show & Aborted & Completed \\
\hline
\end{tabular}

Transforming plain text (C-CDA) to coded values (FHIR) is hard, because first the meaning of a character or combination of characters has to be defined, after which the combination of codes to be used has to be established. This particular example with transforming reference ranges only occurs due to the restrictions of C-CDA, but it serves well as an example to show the difficulties that might occur when one standard represents information in plain text, while another uses coded values.

\subsection{Different Relational Structures/Hierarchies}

Some DCMs have a different structure and hierarchy than their representation. One of the best examples for this is Alert. The DCM of alert is shown in Figure 5, the structure of the representation of the Alert DCM when modeled in FHIR can be viewed in Figure 6 .

The DCM complies with the model used in C-CDA as they are both based on the same RIM, because the alert DCM has been inspired by the Continuity of Care Record (CCR) and Continuity of Care Document (CCD), which are both constraints of CDA. So clearly, the model the DCM author had in mind has influenced the design of the DCM, failing the basic premise that a DCM is technologyagnostic.
In FHIR, alert is modeled slightly differently, as a stand-alone resource.

It contains a category and a note. It can however also have one or multiple extensions (like all FHIR resources). So, if an alert refers to a condition (MRSA) or an allergy/intolerance, one can extend the resource with a resource reference to a condition, allergy, contraindication or any other resource.

So there are clearly some differences between the two structures:

In the DCM, the alert concept directly refers to the reaction and its criticality. This criticality can be mapped to FHIR on the severity of the Allergy or on the severity of the symptom of the adverse reaction. The latter is the one to choose, however, some confusion may easily arise here.

The same goes for the BeginDateTime attribute of an alert in the DCM, which is defined as:

"The date and time the allergy, the adverse reaction or the warning has been set as an Alert" 14

The problem is where to specify this date in the FHIR representation. The alert does not have a date attribute, the allergy has an attribute for the date the allergy is recorded, and the adverse reaction has an attribute which defines the date the reaction began and exposure has an attribute for the initial date of the exposure that is sus-

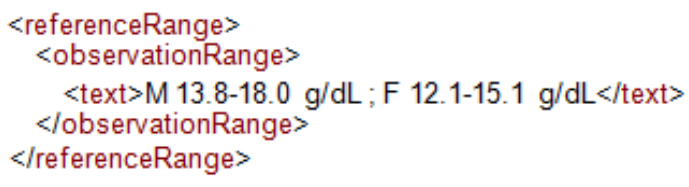

Figure 3: A normal range of hemoglobin represented according to C-CDA. 
pected to be related to the reaction. None of those will actually do. You could however make another extension to alert which specifies the date the alert has been recorded.

These problems are a result of the fact that the technology-independent model and the technical model both have different structures and hierarchies, and therefore some attributes might have a slightly different meaning in both models.

\subsection{Requirements and restrictions}

Different standards have different restrictions and different requirements. For example: A FHIR resource could define an attribute as mandatory, while the DCM or CDA does not. This could form a problem with the transformation: When one transforms a message which lacks an attribute that is mandatory in the target representation, the resulting message will be invalid. The same goes when transforming a message to a representation in which the cardinality of an attribute is lower.

It can even be argued whether the representation of the DCM is still valid if the DCM does not define an attribute as mandatory, while FHIR or CDA does.

For example, we encountered a problem with the total score of the Barthel Index. In FHIR, we used another question in the questionnaire to define this, the questionnaire defines the total score as an answer and the interpretation of the total score in a text attribute. In CDA the total score can be defined in the top of the Assessment Scale Observation, however, there is no attribute to define the interpretation of the score. The best solution, however not ideal, is to add a reference range to define the interpretation.

The difference in requirements and restrictions between CDA, the DCMs, and FHIR also posed a problem when transforming the CDA representation of the DCMs to FHIR. More restrictions in FHIR can result in loss of meaning, while more requirements enforce manual addition of information to the FHIR representation. We encountered such a problem during the transformation of a care plan. A care plan consists of one or multiple components. According to the DCM, these four components are allowed: Encounter, Medical devices Medicine, Vaccination Activity, Other.

Whereas the DCM only requires a reference to the encounter [14] (as does the C-CDA implementation guide [15]), FHIR in addition requires an encounter state and an encounter class, which cannot always be extracted from the CDA representation, because it is not mandatory. Consequently, these attributes must be added manually to the FHIR representation.

\subsection{Narratives}

Both CDA and FHIR enable addition of narratives to messages. In both standards reference is possible to coded entries in a document. This is a similarity that immediately forms a problem when transforming one representation into the other. The problem arises when one entry in CDA becomes two entries in FHIR. This would require a

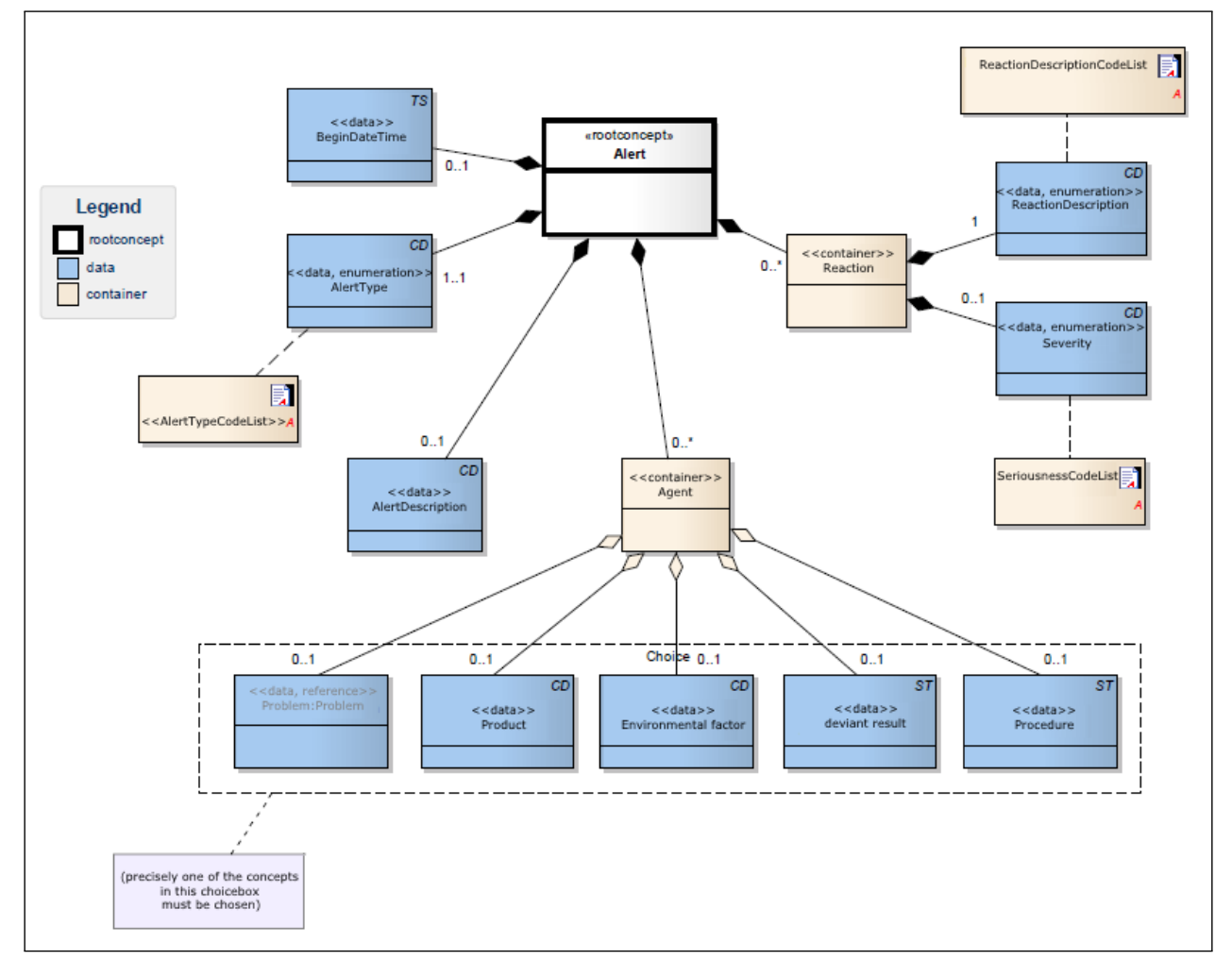

Figure 5: The Detailed Clinical Model of an Alert (remodeled in English for readability) [14. 
choice to which entry the narrative refers. When choosing to refer to both, it is hard to define what the reference means. It could mean that the narrative describes a combination of both entries or that it describes two totally separate entries.

Furthermore, the syntax in which the narrative is defined differs: CDA defines its own XML syntax for narrative content, loosely based on HyperText Markup Language (HTML). FHIR makes use of a constrained set of Extensible HyperText Markup Language (XHTML) which is somewhat more expressive than the CDA markup. [16] This means we would have to transform not only the content and references to the entries, but also the syntax.

\subsection{Null flavors and negation indicators}

In healthcare, it's quite common for data to be unknown, unavailable, have an exceptional value or otherwise fall outside the bounds of a "normal" value. To deal with this, CDA uses the concept of "null flavors", i.e., the different meanings of null values. Examples are: "Unknown", "Not asked", "Positive infinity", "Trace amount", "Masked", and "Other". Null flavors are used on almost every attribute and data type property in its models. Unless an element is explicitly marked as "mandatory", which means no null flavors are permitted, these null flavors can appear anywhere. 4] One example is shown in Figure 7, which represents that the quantity of a maximum dosage of a certain medication is unknown.

$$
\begin{array}{|c|c|}
<\text { maxDoseQuantity nullFlavor="UNK"> } \\
<\text { numerator nullFlavor="UNK"/> } \\
\text { <denominator nullFlavor="UNK"/> }
\end{array}
$$

Figure 7: Example of null flavors in CDA.

FHIR handles null flavors exactly opposite to CDA. In FHIR use of a null flavor must be explicitly allowed, whereas in CDA it is allowed by default, by defining null flavors in the core specification and constraining them (using a specific value set) to those relevant to a specific element.

The same goes for negation indicators. In CDA one can add a negation indicator on almost any act. For example: a negation indicator could specify that a patient was not given a certain medication. In FHIR negation indicators can be added only on places where they are explicitly allowed. In the FHIR specification of the medication administration for example, specific attributes are added to indicate if and why a medication was not given. An example can be found in Figure 8.

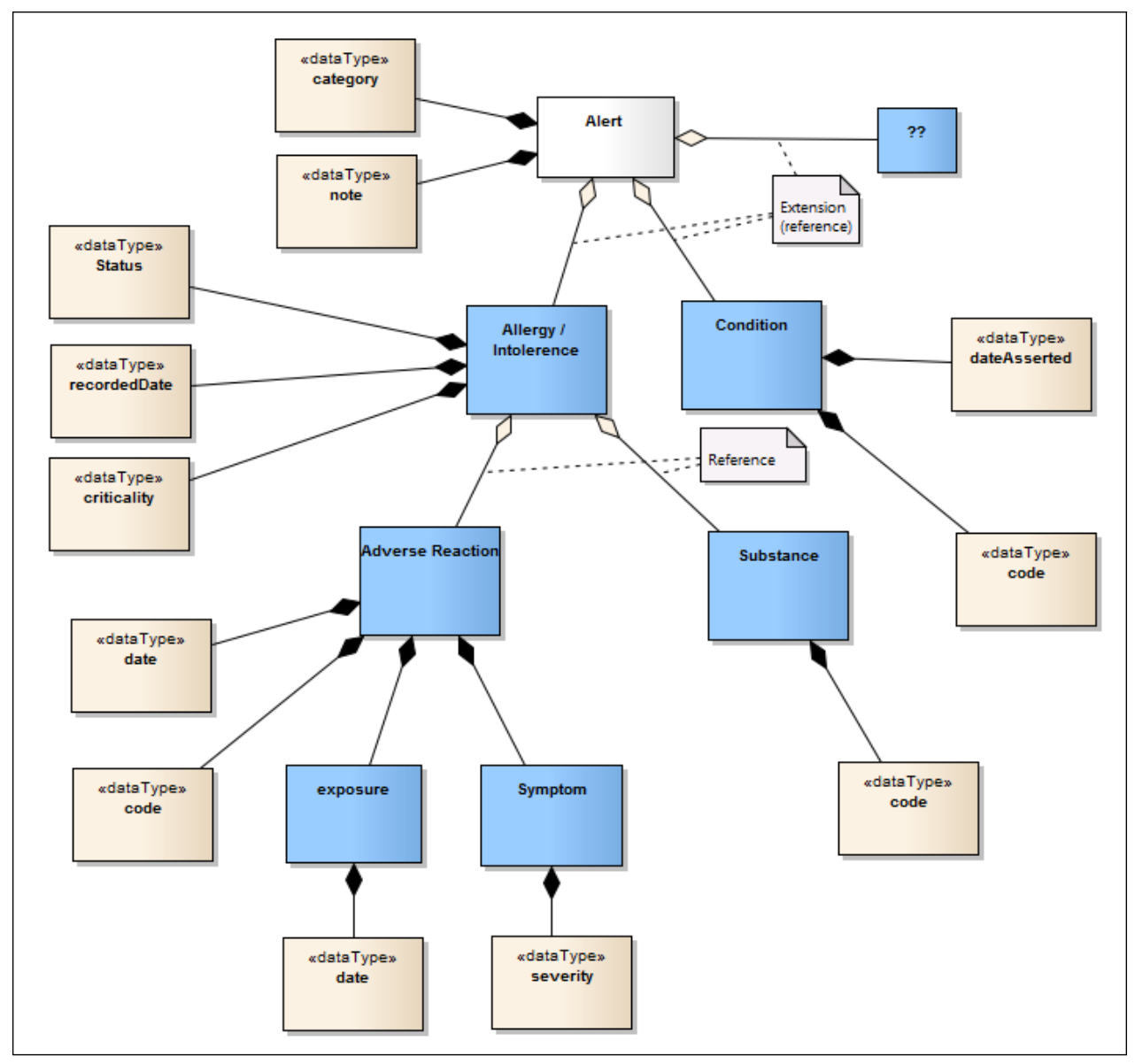

Figure 6: Representation of the alert DCM when modeled in FHIR. 
Because CDA allows null flavors and negation indicators almost everywhere and FHIR allows them only on specific elements, it is difficult to transform a CDA document into a FHIR document without loss of meaning. A solution might be to use extensions for this, however most FHIR developers would not expect these extensions.

\subsection{Meaning of attributes}

Some attributes in FHIR and CDA look similar, but can have a slightly different meaning or a meaning which can be discussed. Because of this difference or vagueness, these attributes are difficult to transform from one representation to another.

For example, CDA uses one general EffectiveTime attribute to specify dates and times in AllergyIntolerance. To create an AllergyIntolerance section in a CDA document a couple of these date attributes are mandatory:

- The effective time in the section which contains all of the patient's allergies and intolerances.

- The effective time of each separate allergy or intolerance.

However, the C-CDA implementation guide [15] does not specify the exact meaning of these effective time attributes, e.g., whether the first descriptions means the time that the first allergy or intolerance was identified. Although probable, this is not explicitly stated. Because of this vagueness, we cannot be sure how to represent this date in a FHIR message. The effective time of each separate allergy and intolerance is probably the time the allergy has been recorded, and therefore can be transformed to the recordedDate attribute in a FHIR message.

\section{Discussion}

Most aspects of GenOGeg's DCMs can be properly represented in both FHIR and CDA, and can be transformed from CDA to FHIR. However, in our study some fundamental problems arose, which could trouble a proper implementation of two standards based on the same DCM. Creating the CDA and FHIR representations of the DCMs shows that combining or mapping different standards could result in several conflicts. The transformation of the CDA representation to FHIR confirms these conflicts and adds several others to the list. Problems we encountered refer to the following aspects:

- Coded values

- Relational structures / Hierarchies

- Requirements and restrictions

- Narrative

- Null flavors and negation indicators

- Meaning of attributes

All problems in these aspects result in either loss or slight change of meaning, and fundamental difficulties during the implementation of the standards and when transforming one standard to the other.

This study shows that DCMs are not technologyindependent, i.e., not every representation of a DCM is necessarily interconvertible with others. Therefore, to allow the implementation of multiple technical models in a DCM, modelers should anticipate on the technological models to be used when defining the DCMs.

As we had to create the CDA example messages ourselves, the definitive GenOGeg CDA messages may slightly differ from the messages that were developed for this study.

We only transformed our messages from CDA to FHIR, not in reverse direction. However, we are quite certain this would not have resulted in fundamentally more problems, as mapping of the representations involves bidirectional analysis and comparison.

The selection of the DCMs was done by one individual, which may have caused selection bias. However, because of the amount of complexity of the selected DCMs we are fairly certain that the majority of the possible problems have arisen from the selected DCMs.

A strength of this study is that we used an XSLT transformation to back up our conceptual analyses through representing the DCMs in both standards. The transformation also identified problems that would not have

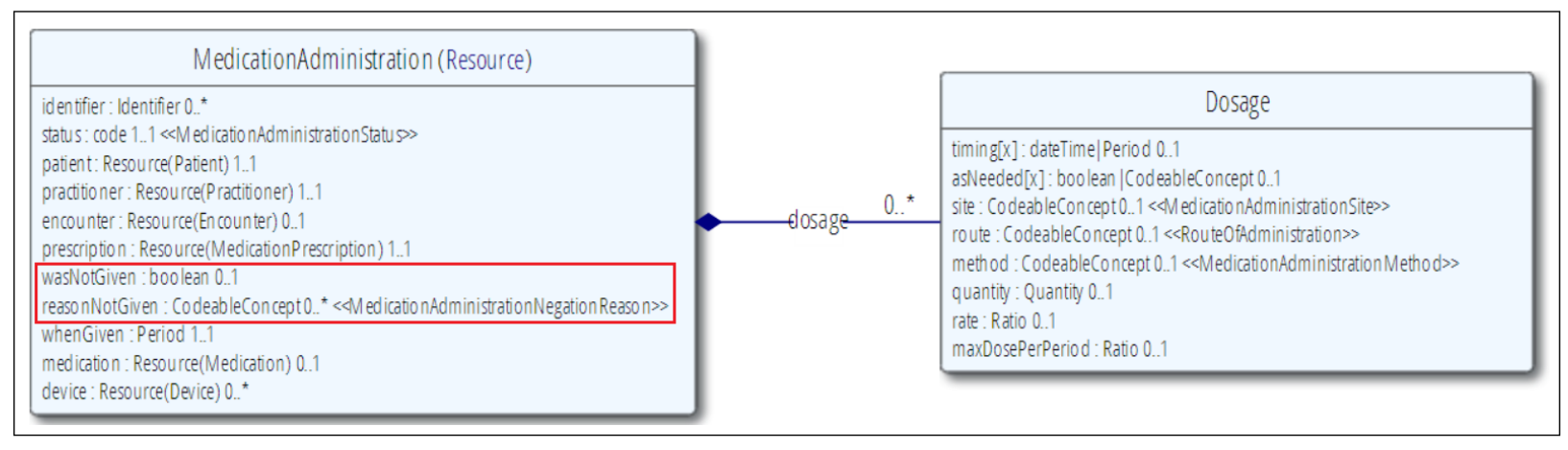

Figure 8: FHIR specification of the medication administration resource [8]. 
arisen otherwise. Even more, all problems identified by the creation of example messages were also identified by the transformation. As both the CDA and FHIR representations are defined in XML, and XSLT is designed to transform XML documents into other XML documents, the choice to use XSLT instead of other methods was a natural one.

Although there is research on mapping or combining two standards, this study is to our knowledge the first to determine if two standards based on the same DCM are interconvertible and retain meaning. Other studies [3, 9, 10, 11] concluded that only small problems arise when mapping or combining two standards, and leave the bigger problems untouched. Goossen et al. identified that HL7 templates and 13606 archetypes object models can be compared on nine different levels [3], but only describe problems in the first 4 . Our study shows that fundamental problems occur on the levels that Goossen et al. do not describe.

The conclusions of our study disagree with the findings of earlier studies as we conclude that with the different procedures and techniques and a broader scope we find several fundamental and unresolvable problems. Actually implementing conversions forced us to face problems that will arise. A paper by Blobel et al. 17 agrees that existing modeling approaches show fundamental weaknesses and differences in maturity and are not all capable to represent the same information.

Because the study is based on a real-life use case (GenOGeg) it can be very useful for its decision makers. The problems we identified are generic and therefore could also be useful for similar projects. The study shows that the GenOGeg's current DCMs are not fully compliant with multiple standards, which is relevant information for both the decision makers working with Nictiz and the academic hospitals, and the active community using detailed clinical models. We hope this study will encourage modelers to take the possibility of the implementation of multiple standards into account when defining future detailed clinical models.

Because FHIR is still in DSTU major revisions in the FHIR standards can still be made. This study could be input into the standard formation process of FHIR, especially in the area where interoperability with other standards is involved.

Using other standards (OpenEHR, ISO-13606, RDF etc.) in addition to FHIR and CDA could give new insights in which problems arise when combining multiple standards based on the same DCMs.

Because the demand of standards that can exchange information with other standards grows, research needs to be done to determine if the current DCM approach needs to be revised to allow for implementation of multiple standards.

\section{Conclusions}

Different representations of a DCM do not necessarily convey the same meaning. In our study we showed that both CDA and FHIR are not fully compliant with each other and with GenOGeg's detailed clinical models when it comes to restrictions and requirements, coded values, relational structures, narrative, null flavors and negation indicators and meaning of attributes. This results in possible loss of meaning and lack of interconvertibility when implementing two separate standards based on the same DCMs. This indicates that it does matter which technical standard is used to implement a DCM.

\section{List of Abbreviations}

$\begin{array}{ll}\text { C-CDA } & \text { Consolidated Clinical Document Architecture. } \\ \text { CCD } & \text { Continuity of Care Document. } \\ \text { CCR } & \text { Continuity of Care Record. } \\ \text { CDA } & \text { Clinical Document Architecture. } \\ \text { DCM } & \text { Detailed Clinical Model. } \\ \text { DSTU } & \text { Draft Standard for Trial Use. } \\ \text { EHR } & \text { Electronic Health Record. } \\ \text { FHIR } & \text { Fast Health Interoperable Resources. } \\ \text { GenOGeg } & \text { Generic Data for Patient Transfers. } \\ \text { HL7 } & \text { Health Level Seven. } \\ \text { HTML } & \text { HyperText Markup Language. } \\ \text { MRSA } & \text { Methicillin-resistant Staphylococcus aureus. } \\ \text { Nictiz } & \text { National IT Institute for Healthcare in the } \\ & \text { Netherlands. } \\ \text { RIM } & \text { Reference Information Model. } \\ \text { XHTML } & \text { Extensible HyperText Markup Language. } \\ \text { XML } & \text { Extensible Markup Language. } \\ \text { XSLT } & \text { Extensible Stylesheet Language Transformations. }\end{array}$

\section{References}

[1] Smeele F. Begeleidend document producten generieke overdrachtsgegevens. Nictiz, may 2013. (Dutch)

[2] Detailed clinical models. Health Level 7 [Internet]. 2013 [accessed 2014 Jan 14]. Available from: http://wiki.hl7.org/ index .php?title=Detailed_Clinical_Models\&oldid=66663

[3] Goossen WT, Goossen-Baremans A. Bridging the hl7 template 13606 archetype gap with detailed clinical models. Stud Health Technol Inform. 2010;160(2): 932-936

[4] Comparison of fhir and v3. FHIR [Internet]. 2014 [accessed 2014 Jun 8]. Available from: http://www.hl7.org/implement/ standards/fhir/comparison-v3.html

[5] Goossen WT, Goossen-Baremans A, van der Zel M. Detailed clinical models: A review. Healthcare Informatics Research. 2010;16(4):201-214

[6] Introducing HL7 FHIR. FHIR [Internet] 2012 [accessed 2013 Jan 13]. Available from: http://www.hl7.org/implement/ standards/fhir/summary.html

[7] FHIR Overview. FHIR [Internet] 2012 [accessed 2014 Jan 13]. Available from: http://www.hl7.org/implement/standards/ fhir/overview.html

[8] FHIR Specification. FHIR [Internet] 2012 [accessed 2014 Feb 11]. Available from: http://www.hl7.org/implement/ standards/fhir/index.html 
[9] Bosca D, Marco L, Burriel V, Jaijo T, Milln JM, Levin A, Pastor O, Robles M, Maldonado JA. Genetic testing information standardization in hl7 cda and iso 13606. MEDINFO 2013, IOS Press, 2013.

[10] Browne E, openEHR Arechtypes for HL7 CDA Documents. OpenEHR Wiki [Internet] 2008 [accessed 2014 april 30] Available from: http://www.openehr.org/wiki/display/ stds/openEHR+Archetypes+for+HL7+CDA+Documents

[11] Moner D, Moreno A, Maldonado JA, Robles M, Parra C. Using archetypes for defining cda templates. Quality of Life Through Quality of Information. IOS Press, 2012.

[12] XSL Transformations. W3 [Internet] 2003, [accessed $2014 \mathrm{Feb}$ 11]. Available from: http://www.w3.org/TR/xslt

[13] Kay M. XSLT 2.0 and Xpath 2.0 Wiley Publishing, Inc. 4th Edition
[14] Klinische Bouwstenen. Nictiz [Internet] 2013[accessed: 2014 Mar 28]. Available from: http: //www.nictiz.nl/page/Expertise/Specialistische-zorg/ Generieke-overdrachtsgegevens/Klinische-bouwstenen (Dutch)

[15] HL7 Implementation Guide for CDA Release 2: IHE Health Story Consolodation, DSTU Release 1.1 Health Level Seven, Inc, 2012 .

[16] Comparison of FHIR and CDA. FHIR [Internet] 2014 [accessed 2014 May 9]. Available from: http://www.hl7.org/implement/ standards/fhir/comparison-cda.html.

[17] Blobel B, Goossen W, Brochhausen M. Clinical modeling - a critical analysis. International Journal of Medical Informatics. 2014;83:57-69. 